\title{
Was kann moderne Linguistik für die Fremdsprachendidaktik leisten? Ausgewählte Aspekte
}

\author{
What can modern linguistics do \\ for foreign language didactics? Selected aspects
}

Adam SZELUGA ${ }^{1}$

Uniwersytet Gdański

\begin{abstract}
The article deals with the most important relations between Foreign Language Didactics and the main theoretical models in modern linguistics, especially the Applied Linguistics of second- and foreign-language teaching. Theories and models of modern linguistics have often laid the theoretical foundations of foreign language teaching, as we can observe in the individual methods and learning techniques (from structuralism to generative grammar, communicativepragmatic turn of the 60s and 70s, cognitive linguistics and to F. Grucza's anthropocentric theory of languages). In this perspective, the purpose of this article is to raise and discuss the question of how modern linguistic theories can improve the effectiveness of language teaching.
\end{abstract}

Keywords: Foreign language teaching, glottodidactics, models of glottodidactics scheme, linguistics, language system, methodology, universalism, relativism, cognitive theories, the anthropocentric theory of languages

\section{Zusammenfassung}

Im Artikel werden die wichtigsten Relationen zwischen der Fremdsprachendidaktik und der Linguistik, insbesondere der angewandten Linguistik behandelt. Linguistische Theorien und Modelle waren oft theoretische Grundlagen des Fremdsprachenunterrichts, was in einzelnen Methoden und Lerntechniken sichtbar wurde (vom Strukturalismus, über generative Grammatik,

1 (D) https://orcid.org/0000-0002-2196-6125. 
kommunikativ-pragmatische Wende der 60-er und 70-er Jahre, kognitive Linguistik bis zur anthropozentrischen Theorie der Sprachen von F. Grucza). In dieser Perspektive werden ausgewählte Möglichkeiten dargestellt, wie moderne linguistische Theorien vor allem die Effektivität des Fremdsprachenunterrichts verbessern können.

Keywords: Fremdsprachenunterricht, Glottodidaktik, Modelle des Glottodidaktikschemas, Linguistik, Sprachsystem, Methodik, Universalismus, Relativismus, kognitive Theorien, anthropozentrische Sprachtheorie

\section{Einführung}

Fremdsprachendidaktik ist als Teildisziplin der angewandten Linguistik in den 50-er und 60-der Jahren des 20. Jahrhunderts entstanden und wird heute von den meisten Forschern als eine völlig autonome wissenschaftliche Disziplin angesehen (u.a. Pfeiffer, 2001, S. 13-19; Grucza, 2007, S. 311-322; Skowronek, 2013, S. 9-23; Dakowska, 2014, S. 28-38), auch wenn ihr Forschungsgegenstand, Ziele und Methoden besonders interdisziplinär angelegt sind. Jedoch unter allen verwandten Wissenschaften gibt es eine, deren Relevanz für die Entwicklung gegenwärtiger Fremdsprachendidaktik nicht zu überschätzen ist. Es ist die Sprachwissenschaft, die als Oberbegriff für alle Untersuchungen verstanden wird, die Sprache als solche zu ihrem Gegenstand gemacht haben. Auf moderne sprachwissenschaftliche Forschungen, besonders auf die seit der Mitte des 20. Jahrhunderts, wird der Terminus Linguistik bezogen. Zwischen der Fremdsprachendidaktik und der Sprachwissenschaft (darunter auch Linguistik) bestanden immer sehr enge wechselseitige Relationen. Linguistische Theorien und Modelle dienten oft als theoretische Grundlagen des Fremdsprachenunterrichts, was vor allem in einzelnen Methoden und Lerntechniken sichtbar wurde.

Das Hauptziel dieses Beitrags versteht sich als ein Versuch, die Korrelation zwischen den wichtigsten sprachwissenschaftlichen Theorien und Ansätzen (v.a. den der letzten 20-30 Jahre) und den Entwicklungstendenzen der Fremdsprachendidaktik zu schildern (vom Strukturalismus, über generative Grammatik, Glottokodematik, kommunikativpragmatische Wende der 60-er und 70-er Jahre, kognitive Linguistik bis zur anthropozentrischen Theorie der Sprachen von F. Grucza). 


\section{Wissenschaftlich fundierte Methoden des Fremdsprachenunterrichts im historischen Abriss}

Geschichte des Fremdsprachenunterrichts ist nicht viel kürzer, wie die Geschichte der Menschheit selbst, weil die Kenntnis von Fremdsprachen eine gelungene Kommunikation zwischen Vertretern verschiedener Nationen ermöglichte. Bekannte Überlieferungen über ein institutionell organisiertes Fremdsprachenlernen stammen aus dem Summer, Ägypten und dem antiken Rom und sind ein paar Tausend Jahre alt. Doch von ersten mindestens teilweise wissenschaftlich gestützten Methoden des Fremdsprachenunterrichts kann man erst seit der Zeit der Aufklärung und des Barocks sprechen, wo man fast ausschließlich Latein und andere klassische Sprachen gelehrt hat. Damalige sog. Philologische Methode basierte auf den Übersetzungstechniken (aus dem Original) und der Erklärung einzelner Grammatikstrukturen (kontrastiv, aus der Sicht der Muttersprache der Schüler), wobei hier zu bemerken ist, dass sich die bekanntesten Didaktiker, wie Erasmus von Rotterdam, Wolfgang Ratke oder Jan Amos Komensky über Vorteile und Nachteile der Deduktion und der Induktion als der führenden Lehrtechnik auseinandergesetzt haben (Ronowicz, 1982, S. 9-15). Diese Diskussionen können zweifellos als die Grundlage für die spätere methodologische Eingrenzung der Methoden in direkte (ohne Einsatz der Muttersprache) oder indirekte (zweisprachige) angesehen werden.

Erste Methode, die nicht nur auf Erfahrung und Intuition der Lehrer fußte (wie z. B. die Natürliche Methode), war die GrammatischÜbersetzungsmethode von J. V. Meidinger. In seiner 1783 herausgegebenen Grammatik der französischen Sprache hat er jede sprachliche Regel mit entsprechenden Kontextbeispielen verbunden, was heute als deduktivinduktive Technik bekannt ist. Seine Nachfolger, J. H. Seidenstücker (1811), Karl Plötz (1848) und H. S. Ollendorf (1864) haben in ihren Lehrwerken Elemente der kontrastiven Grammatik und vereinfachte Ausspracheregeln eingesetzt. Sichtbar wurde auch eine deutliche Bereicherung der Übungstechniken um freie Sprechübungen, weil frühere Methoden einen überwiegend schriftlichen Charakter hatten. Ein interessantes Beispiel in diesem Zusammenhang kann die Serienmethode von F. Gouin (1892) sein, die das Sprechen mit dem Handeln in Verbindung gesetzt hat und im gewissen Sinne als Vorläufer der späteren Sprechakttheorie von Austin/Searle gesehen werden kann (Ronowicz, 1982, S. 56-85). Indirekte Methode von M. D. Berlitz (1878) wendet sich auch dem natürlichen Spracherwerb zu und ihr Autor lenkt seine Aufmerksamkeit auf die praktische Seite der Sprachbeherrschung. 
Weitere Schritte in der Entwicklung der Fremdsprachendidaktik weisen schon konkrete Zusammenhänge mit den sich entwickelnden sprachwissenschaftlichen Theorien auf und können deshalb als eindeutig wissenschaftlich untermauert bezeichnet werden.

\section{Neusprachliche Reformbewegung und strukturalistische Ansätze in der Fremdsprachendidaktik}

Im Jahre 1882 hat Wilhelm Viëtor, der bekannte deutsche Sprachwissenschaftler und Vertreter der neusprachlichen Reformbewegung gegen Ende des 19. Jahrhunderts, seine Auffassungen über das Fremdsprachenlernen im Werk „Der Sprachunterricht muss umkehren“ ausgelegt. Den Schwerpunkt im Lernprozess legt er auch auf die mündlichen Sprachfertigkeiten, doch im Gegensatz zu früheren Forschern schreibt er viel mehr Bedeutung der Phonetik zu. Er war 1896 einer der Mitbegründer der Internationalen Phonetischen Gesellschaft (IPA), die das heute bekannte Transkriptionssystem geschaffen hat. Qualitative und quantitative Lautbeschreibung war eines der ersten Elemente des Sprachsystems, dessen Erforschung eine praktische Verwertung in der Unterrichtspraxis fand und das die Qualität der Aussprache im Fremdsprachenunterricht entschieden verbessert hat. Ihre Fortsetzung und Erweiterung findet man in der ersten Hälfte des 20. Jahrhunderts vor allem in der Prager Schule des Strukturalismus.

Die Grammatikvermittlung hat sich auf die Grammatik der Muttersprache und nicht auf die lateinische Grammatik bezogen und man hat sie induktiv, in ausgewählten kommunikativen Kontexten dargeboten. Diese pragmatische Ausrichtung des Fremdsprachenunterrichts hat allerdings nicht immer bahnbrechende Wirkung gehabt. Erst in den 60-er Jahren des 20. Jahrhunderts wurde es möglich, auch pragmatische Aspekte zu berücksichtigen. Es geschah im Rahmen der audiolingualen Methode, die behavioristisch ausgerichtet war (Roche, 2005, S. 14-15). Die Nachfolger von Viëtor, H. Sweet, O. Jespersen und H. Palmer haben als die ersten Sprachdidaktiker mit Hilfe der strukturalistischen Kategorien und Subkategorien einzelne Lernstufen bestimmt. Wichtigste Lernziele wurden dabei an kommunikative Bedürfnisse der Schüler angepasst. Man hat auch reale Sprachvarietäten unterrichtet, darunter die Umgangssprache. Diese Forscher gelten außerdem als Autoren der meisten grammatischen und lexikalischen Übungen, Lerntechniken und Lernstrategien, die bis heute aktuell geblieben sind (Szeluga, 2008, S. 10). 
Die von F. de Saussure getroffene Unterscheidung zwischen der Sprache als einem theoretischen System (la langue) und ihrer konkreten Realisierung vom jeweiligen Sprecher (la parole) hat geholfen, viel genauere Lernziele im Fremdsprachenunterricht zu formulieren, und zwar auf der Ebene aller vier Sprachfertigkeiten: Hörverstehen, Lesen, Sprechen und Schreiben. Sprachstoff sowie Unterrichtsmaterialien konnten dabei so gestaltet werden, dass sie eine entsprechende Lernstoffprogression garantierten. Grammatische und lexikalische Komponenten wurden in funktionalen Einheiten präsentiert, die einen weiten kommunikativen Kontext (v.a. Satz) umfassten.

Die Kontroversen zwischen der direkten und der GrammatischÜbersetzungsmethode hatte auch 1925 der polnische Germanist, Sprachdidaktiker und Lehrbuchautor Juliusz Ippoldt aufgegriffen. Sein Vorschlag war die sog. vermittelnde Methode, die einen holistischen Charakter hatte und von der gesprochenen Sprachvariante ausging. Ippoldt sah ähnlich, wie die Vertreter der Reformbewegung einen großen Wert sowohl in der Phonetik und der phonetischen Umschrift als auch in der Vermittlung ganzer kommunikativer Kontexte. Der Unterschied lag vor allem in der Grammatik, welcher er eine viel bedeutendere Rolle im Unterrichtsprozess beigemessen hat (Ronowicz, 1982, S. 149-154).

\section{Angewandte Linguistik und moderne Fremdsprachendidaktik}

In vielen modernen sprachwissenschaftlichen Forschungen wird bekanntlich, wie schon oben bemerkt wurde, zwischen zwei Begriffen unterschieden: ,Sprachwissenschaft' und ,Linguistik‘. Eine spezielle Rolle wird bei dieser terminologischen Abgrenzung einer relativ neuen Subdisziplin der Linguistik eingeräumt, der angewandten Linguistik, für deren Entwicklung vor allem die letzten 50-60 Jahre von Bedeutung waren. Man könnte allerdings in diesem Zusammenhang auch auf einige frühere Untersuchungen verweisen, die der Sprachwissenschaft einen mehr praktisch und empirisch ausgerichteten Charakter gegeben haben.

Im Jahre 1803 veröffentlichte August Ferdinand Bernhardi in Berlin den zweiten Teil seines Werkes Sprachlehre, nämlich Angewandte Sprachlehre. Somit hat er reine und angewandte Sprachwissenschaft als zwei Anwendungsbereiche der Sprache in der Poesie und Wissenschaft genannt. Ein wichtiger Vorläufer der heutigen angewandten Linguistik war J. Baudouin de Courtenay, der nicht nur praktische Ausrichtung der Sprachwissenschaft, 
sondern auch ihre Bedeutung für andere wissenschaftliche Disziplinen anerkannt hatte. Eine ähnliche Auffassung haben auch solche europäischen Forscher vertreten, wie z. B. Aleksandr I. Tomson (1910), Theodor Steche (1925), Eugen Wüster (1931), B. Migliorini oder G. Kandler (1952) (Grucza, 1983, S. 342-358; Grucza, 2007, S. 45-48). Einen grundlegenden Baustein für angewandte Linguistik hat jedoch die amerikanische Linguistik der 40-er und 50-er Jahre gelegt. 1946 führte Ch. C. Fries angewandte Linguistik als Studienfach an der Universität in Michigan ein und damit hat er diese Subdisziplin der Linguistik als selbständige wissenschaftliche Disziplin fundiert. Im Jahre 1950 wurde erste Zeitschrift für angewandte Linguistik gegründet (Language Learning, a Journal of Applied Linguistics) und 1959 entstand erstes Institut für Angewandte Linguistik (Washington). Primärer Forschungsgegenstand der angewandten Linguistik war die weit verstandene Problematik des Fremdsprachenerwerbs und der Fremdsprachendidaktik. Im Laufe der Zeit haben sich diese Forschungen wesentlich ausgebreitet und heutzutage könnte man viele weitere Wissenschaftsbereiche und zum Teil auch autonome wissenschaftliche Teildisziplinen als zentrale Fragen der angewandten Linguistik nennen.

\section{Konstituierung der polnischen Glottodidaktik}

Als Vater der polnischen Glottodidaktik kann ohne Zweifel Ludwik Zabrocki bezeichnet werden. Er bediente sich zwar der Termini Methodik und Didaktik der Fremdsprachen, doch von Anfang an sah er einen engen Zusammenhang zwischen diesen Untersuchungsbereichen und der Linguistik, und eben nicht der Pädagogik. Auf diese Art und Weise hatte er linguistische Grundlagen für spätere methodologische Fundierung der Glottodidaktik geschaffen (Grucza, 2007, S. 311-312). Außer Ludwik Zabrocki gilt auch Waldemar Pfeiffer, sein Schüler an der Adam-Mickiewicz-Universität in Poznań, als einer der Väter der polnischen Schule der Glottodidaktik. Unter anderem dank ihm hat sich positiv die entscheidende Frage geklärt, ob diese Forschungen als eine autonome wissenschaftliche Disziplin, mit allen ihren konstitutiven Bedingungen anerkannt werden können.

Eine nächste Erweiterung ihrer Forschungen hat die polnische Glottodidaktik am Institut für Angewandte Linguistik der Warschauer Universität erfahren. Unter Führung von Franciszek Grucza und seinen Mitarbeitern wurde in den 70-er und 80-er Jahren ein umfangreiches methodologisches Modell ausgearbeitet, das die neu fundierte Disziplin, Glottodidaktik, zu einer immanenten Konstituente der heutigen angewandten 
Linguistik gemacht hat. Als Hauptprobleme gelten dabei Fragen des Spracherwerbs (muttersprachliche und fremdsprachliche Perspektive) mit allen Elementen des sog. glottodidaktischen Gefüges: Lehrer, Schüler, Methoden, glottodidaktische Materialien, Lernbedingungen mit der Sprachenpolitik des Staates.

Moderne Glottodidaktik ist daher eine äußerst interdisziplinär angelegte wissenschaftliche Disziplin. Ihre Problematik beginnt bei rein linguistischen Fragen der Sprache selbst und ihres Wesens, und umfasst auch die neuround psycholinguistischen Gebiete des Spracherwerbs, Sprachdidaktik aus pädagogischer Perspektive, Sozio- und Pragmalinguistik, Literaturdidaktik, interkulturelle Studien, bis hin zur Computerlinguistik, um nur die wichtigsten Disziplinen zu nennen (vgl. u.a. Pfeiffer, 2001, 28-31).

Unter linguistischen Teildisziplinen jedoch, die für diesen Beitrag besondere Bedeutung haben und moderne Glottodidaktik determinieren, könnte man folgende nennen:

$\begin{array}{cl} & \text { Neurolinguistik } \\ & \text { Psycholinguistik } \\ & \text { Soziolinguistik } \\ & \text { Kodematik } \\ & \text { Theorien der Kommunikation } \\ & \text { Translatorik (Übersetzungswissenschaft) } \\ \text { Fremdsprachendidaktik } \longrightarrow & \text { Phonologie/Phonetik/Morphologie/Syntax } \\ \text { (Glottodidaktik) } & \text { kontrastive (konfrontative) Linguistik } \\ & \text { Lexikographie } \\ & \text { Lexikologie } \\ & \text { Pragmatik } \\ & \text { Semantik }\end{array}$

Schema 1. Linguistische Subdisziplinen in der Fremdsprachendidaktik

\subsection{Glottokodematik und ihr Platz in der polnischen Glottodidaktik}

Glottokodematik ist als eine Subdisziplin der angewandten Linguistik in Polen entstanden. Ihre Grundlagen hat M. Szczodrowski, einer der Schüler von Ludwik Zabrocki, geschaffen, der die kodematischen Forschungen von Ludwik Zabrocki fortgesetzt und eigene Leistungen mit den glottodidaktischen Theorien verbunden hat.

Szczodrowski definiert in Anlehnung an Zabrocki den Begriff der Sprachmatrizen und erläutert ihre Arten und ihre Bedeutung für die Erlernung einer Fremdsprache aus der Perspektive der allgemeinen Linguistik und der 
Psycholinguistik. In diesem Kontext werden enge Relationen zwischen den linguistischen Theorien und der Glottodidaktik dargelegt. Einen hohen Stellenwert haben in den kodematischen Untersuchungen u.a. die Konstruktion von sprachlichen Materialien, Taxonomie der Lernziele im Fremdsprachenunterricht, die Wahl von Sprachstrukturen (phonologische, morphologische, syntagmatische und syntaktische). Einer der wesentlichen Prozesse des Fremdsprachenerwerbs ist die Steuerung der Kodierung und der Dekodierung von sprachlichen Äußerungen. Diese Prozesse steuert das Rückkoppelungsgefüge, das die entsprechende Kontrolle und Korrektur der anzueignenden Strukturen sichert. Die Herausbildung von Sprachmatrizen entscheidet über kommunikative Kompetenz des Sprechers, was Szczodrowski anhand der kybernetisch-strukturellen Theorie des Fremdsprachenunterrichts erläutert (Szczodrowski, 2009, S. 9-20).

Glottokodematik integriert kodematische und kybernetische Konzepte von Ludwik Zabrocki, erweitert sie weitgehend um neue Komponenten und bringt sie in interdisziplinäre Kontexte. Glottokodematische Forschungen bedeuten einen wesentlichen Fortschritt bei der Erarbeitung einer ganzheitlichen Theorie des Fremdsprachenunterrichts. Dank ihnen wird der vielschichtige, multidisziplinäre Charakter der Lehr- und Lernprozesse einer Fremdsprache äußerst gut ersichtlich.

Potenzielle Möglichkeiten einer Fortsetzung der glottokodematischen Problematik kann man in manchen linguistischen Tendenzen letzter Jahrzehnte, besonders in einigen Theorien der kognitiven Linguistik, bemerken.

\section{Moderne Fremdsprachendidaktik nach dem 2. Weltkrieg}

\subsection{Amerikanischer Strukturalismus und Generative Transformationsgrammatik im Fremdsprachenlernen}

Einen sehr starken Widerhall hat in der modernen Fremdsprachendidaktik der amerikanische Strukturalismus hervorgerufen, dessen Hauptvertreter Ch. C. Fries und J. Bloomfield die sog. Linguistische Methode entwickelt haben, die in den 50-er und 60-er Jahren als Audio-linguale Methode bekannt wurde. In der deskriptiven Analyse aus der synchronischen Perspektive lehnen sie das von F. de Saussure geschaffene universelle Sprachenmodell ab (Grucza, 1983, S. 157-159). Die Audio-linguale Methode stützte sich dabei auf die behavioristischen Ansätze (Stimulus-Reaktion) und ihr lag die Voraussetzung zu Grunde, dass im Fremdsprachenunterricht vor allem 
entsprechende (feste) Gewohnheiten gebildet und automatisiert werden sollen. Mit Hilfe von mehrmaligen Wiederholungsübungen (sog. pattern drills) und durch Nachahmung von Musteräußerungen des Native Speakers verfestigten die Schüler in ihrem Gedächtnis sprachliche Gewohnheiten, die sie dann in einer konkreten kommunikativen Situation wiederrufen konnten. Erste und wichtigste Sprachform ist in dieser Methode die gesprochene Sprache und dementsprechend dominieren im Unterricht solche Fertigkeiten, wie Hörverstehen und Sprechen (Ronowicz, 1982, S. 169-171). Die Audio-linguale Methode hat die Unterschiede zwischen der Muttersprache und der jeweiligen Fremdsprache betonnt, weil fehlerhafte Gewohnheiten aus dem muttersprachlichen System in die zu erlernende Fremdsprache übertragen werden. Als Folge dessen sind kontrastive Studien (kontrastive Linguistik) entstanden, die in der Geschichte der Fremdsprachendidaktik eine bedeutende Rolle spielten.

Viel weniger Einsatz im Fremdsprachenunterricht fanden allerdings die Forderungen der generativen Grammatik, die einen mehr theoretischen Charakter hatten. Die Unterscheidung zwischen Kompetenz und Performanz hat zwar die pragmatische Komponente des Fremdsprachenlernens verstärkt, doch problematisch bleibt die Frage, wie man zu den Regeln der Kompetenz gelangen kann, ohne über die Oberflächenstruktur zu gehen (Zabrocki, 1977, S. 42-43)? Die Tiefenstruktur der Sprache scheint im Fremdsprachenunterricht sehr schwer zu erlernen und in ihr liegt das eigentliche Bedeutungsspektrum, das anzueignen ist.

\subsection{Kommunikativ-pragmatische Wende der 60-er und 70-er Jahre und Kommunikative Methode}

Eine reale Wende in der Fremdsprachendidaktik hat die Kommunikative Methode nach der kommunikativ-pragmatischen Wende der 60-er und 70-er Jahre gebracht, die als eine Art Antwort auf die Sprechakttheorie von Austin/Searle sowie soziolinguistische Untersuchungen (Hymes, Wunderlich, Habermas) betrachtet werden kann. Das Interesse der Linguisten hat sich auf den pragmatischen Aspekt der Sprache, den realen Sprachgebrauch verschoben, d.h. auf das Handeln in der und durch die Sprache. Zentraler Begriff im kommunikativen Ansatz ist die kommunikative Kompetenz, verstanden als die Fähigkeit des Sprechers sich in der jeweiligen kommunikativen Situation erfolgreich $\mathrm{zu}$ verständigen. Kommunikative Kompetenz bezieht sich einerseits auf den Kompetenzbegriff von N. Chomsky, andererseits berücksichtigt sie viel mehr den Performanzbegriff. Der Erwerb 
von Performanz soll das oberste Lernziel im Fremdsprachenunterricht bestimmen, weil nur sie alle Bedingungen einer kommunikativen Situation adäquat wiederspiegelt. Durch den Einsatz von authentischen Texten hat man nicht nur die Motivation, sondern auch die praktische Ebene des Spracherlernens verstärkt. Wesentliche Mängel dieser Methode liegen jedoch in der Grammatikvermittlung, weil ihre Funktionen sehr funktional definiert wurden. Grammatische Korrektheit wurde eindeutig der realen Verständigung untergeordnet und der Sprachfehler war kein Maßstab mehr für die Sprachkompetenz der Schüler (Szeluga, 2008, S. 14-15). Damit verringert sich auch deutlich die Zielgruppe des kommunikativ orientierten Fremdsprachenunterrichts.

\subsection{Aspekte der kognitiven Linguistik in der Glottodidaktik}

Kognitive Forschungen (in der Linguistik, aber auch in den weit gefassten Kognitionswissenschaften) haben nach wie vor einen sehr interdisziplinären Charakter, so dass man keinesfalls nur von einer ganzheitlichen Theorie für die Fremdsprachendidaktik sprechen könnte. Viele Forscher betonen z. B. den holistischen, modularen Charakter der menschlichen Sprachfähigkeiten (Sadownik, 2010).

Es lassen sich allerdings einige Stützpunkte nennen, die gewisse Entwicklungschancen für moderne Glottodidaktik bieten.

Nach der Auffassung der kognitiven Linguistik verarbeitet der Mensch verbale und nonverbale Informationen, indem er sein kognitives Potential in sozialen, kommunikativen Situationen nutzt. Menschliches Gehirn verfügt über entsprechende Strukturen, die mentale Repräsentationen der außersprachlichen Realität bilden und auf diese Art und Weise das ganze Bild der Welt erschaffen. Die Kognitionswissenschaften sind natürlich ein interdisziplinäres Bündel von Wissenschaften, die grenzüberschreitend die Möglichkeiten der menschlichen Erkenntnis ergründen. Für die Glottodidaktik scheinen folgende Begriffe und Konzepte besonders aussagekräftig zu sein: Prototypenbildung (mit der Prototypensemantik), sprachliche Verarbeitungsprozesse und ihr Wesen, Bildung der mentalen Repräsentationen und Kategorisierungsprozesse. Außersprachliche Realität wird mit Hilfe von entsprechenden sprachlichen Mitteln verarbeitet, d.h. konzeptualisiert. Jedes einzelne Sprachsystem entwickelt eigene Konzepte, die diese Wirklichkeit ordnen, systematisieren und wahrnehmen helfen. Der Grad der Konventionalisierung wird bei diesem Prozess zum fundamentalen Mittel des sprachlichen Ausdrucks. Kognitivisten 
unterscheiden zwischen dem individuellen und dem allgemeinen Grad der sprachlichen Konventionalisierung, was den relativistischen Auffassungen ziemlich ähnlich $\mathrm{zu}$ sein scheint. Für Theorie und Praxis des Fremdsprachenlernens kann diese Vorgehensweise neue Chancen eröffnen, z. B. bei der Sprachvermittlung, besonders der Lexikdarstellung, ihrer Vermittlung, Einübung und Automatisierung kann eine balancierte kommunikative Kompetenz des Sprechers erlangt werden:

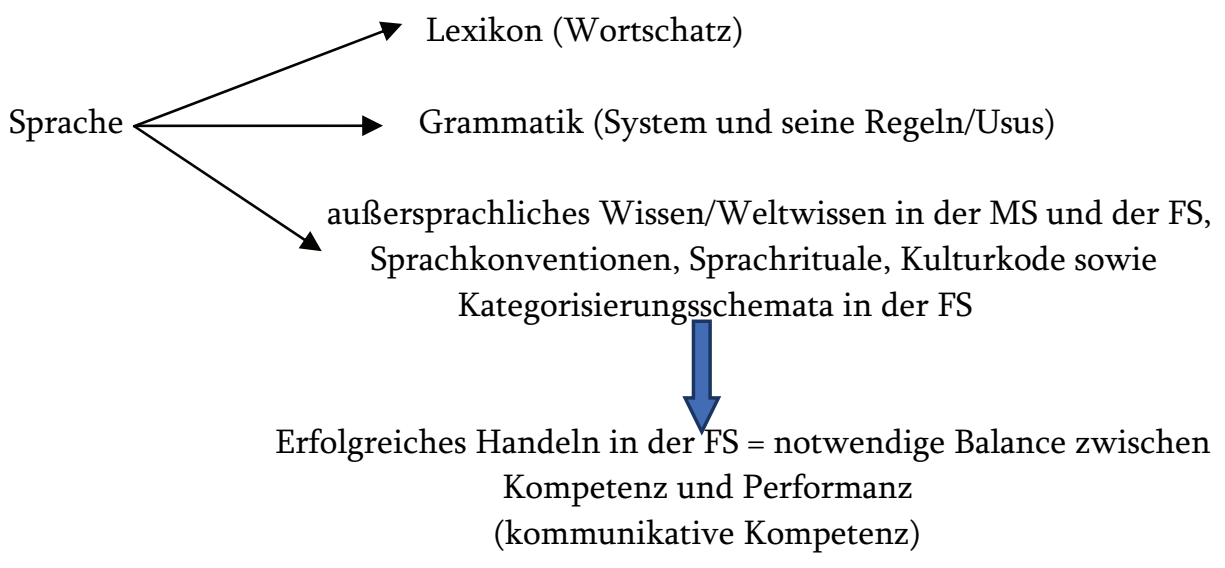

Schema 2. Komponenten der Kompetenz im Fremdsprachenerwerbsprozess

\section{Glottodidaktik und die Anthropozentrische Theorie der Sprachen von F. Grucza}

Die anthropozentrische Theorie der Sprachen von F. Grucza fasst in ihrer Konzeption jeden einzelnen Menschen und somit auch jeden Sprecher mit allen seinen sprachlichen, kognitiven Fähigkeiten auf. Die Sprachbeschreibung kann nach ihr nur aus der Perspektive des einzelnen Menschen geschehen, was bedeutet, dass Grucza alle Modelle ablehnt, die menschliche Sprachen als ideale (damit auch abstrakte) Systeme auffassen. Nur der reale Sprecher, der seine Sprache internalisiert hatte, kann sie gebrauchen. Sprachen existieren also nur als wirkliche und nicht abstrakte Konstrukte, als reale Idiolekte oder ihre Mengen (z.B. Soziolekte) und als solche lassen sie sich charakterisieren und beschreiben (Grucza, 2012, S.19-20).

Sprachliche Fähigkeiten beziehen sich auf das Wahrnehmen und das Verstehen von konkreten Äußerungen einerseits und andererseits ermöglichen sie das Realisieren von diesen Äußerungen im Kommunikationsprozess. Das internalisierte Wissen (sowohl das sprachliche als auch nichtsprachliche, 
deklarative und prozedurale Wissen) ermöglicht jedem Sprecher erfolgreiches Kommunizieren in der Sprachgemeinschaft.

Für die Fremdsprachendidaktik bedeutet es, dass in der Aneignung einer fremden Sprache ganzheitliche und komplexe, kommunikative Fähigkeiten entwickelt werden müssen. Diese Feststellung sollte auch bei der Wahl der Lerninhalte, des Lernstoffs aber auch der Arbeitsformen berücksichtigt werden.

Die Anthropozentrische Theorie der Sprachen von F. Grucza kann bestimmt künftige glottodidaktische Modelle anregen und ihren Platz in der fremdsprachendidaktischen Praxis finden.

\section{Fazit}

Sprachwissenschaftliche und linguistische Modelle haben zweifellos die Entwicklung der Fremdsprachendidaktik weitgehend inspiriert, mitgestaltet und weiterentwickelt. Fast jede linguistische Schule, Tendenz oder Strömung hat neue Inhalte und praktische Resultate für die Theorie und Praxis des Fremdsprachenunterrichts gebracht. Fremdsprachendidaktische Paradigmen lassen sich sehr einfach ihren aktuellen Tendenzen gegenüberstellen, auch wenn dieser Einfluss nicht unbedingt immer und in jeder Methode auf den ersten Blick deutlich zu erkennen war. Sprachlicher Universalismus und Relativismus haben indirekte und direkte Methoden befruchtet und ohne linguistische Determinanten wäre die Geschichte des Fremdsprachenunterrichts so gut wie fast undenkbar und bestimmt viel ärmer. Glottodidaktische Praxis ist dank linguistischen Anregungen nicht nur interessanter, sondern auch effektiver geworden.

Die konstituierende und unverzichtbare Komponente jeder Wissenschaft, auch der Glottodidaktik, ist das Modellieren, sowohl in der Domäne ihrer theoretischen Voraussetzungen als auch hinsichtlich ihrer praktischen Umsetzungen. Aus dieser Sicht bietet moderne Linguistik der Fremdsprachendidaktik zukunftsträchtige Möglichkeiten. Nachfolgende Forschungen mögen hoffentlich neue, erfolgsversprechende Konzepte schaffen.

\section{Bibliographie}

Dakowska, M. (2010). Glottodydaktyka jako nauka. Lingwistyka Stosowana, 3, 71-86.

Dakowska, M. (2014). O rozwoju dydaktyki języków obcych jako dyscypliny naukowej. Warszawa: Wydawnictwa Uniwersytetu Warszawskiego. 
Elsen, H. (2014). Linguistische Theorien. Tübingen: Narr Francke Attempto Verlag.

Grucza, F. (1983). Zagadnienia metalingwistyki. Lingwistyka-jej przedmiot, lingwistyka stosowana. Warszawa: Państwowe Wydawnictwo Naukowe.

Grucza, F. (2007). Lingwistyka stosowana. Historia-Zadania-Osiągnięcia. Warszawa: Euro-Edukacja.

Grucza, F. (2012). Antropocentryczna a paradygmatyczna (tradycyjna) lingwistyka (stosowana) i kulturologia (stosowana). Lingwistyka Stosowana 6, 5-43.

Grucza, S. (2010). Główne tezy antropocentrycznej teorii języków. Lingwistyka Stosowana, 2, 41-68.

Olpińska, M. (2009). Implikacje glottodydaktyczne antropocentrycznej teorii języków. Lingwistyka Stosowana, 1, 187-200.

Pfeiffer, W. (2001). Nauka języków obcych. Od praktyki do praktyki. Poznań: Wagros.

Rickheit, G., Weiss, S., Eikmeyer, H. J. (2010). Kognitive Linguistik. Theorien, Modelle, Methoden. Tübingen: A. Francke Verlag.

Roche, J. (2005). Fremdsprachenerwerb und Fremdsprachendidaktik. Tübingen: A. Francke Verlag.

Ronowicz, E. A. (1982). Kierunki w metodyce nauczania języków obcych. Warszawa: Wydawnictwa Szkolne i Pedagogiczne.

Sadownik, B. (2010). Modulare Architektur der menschlichen Sprachfähigkeit. Kognitive und neurobiologische Perspektive. Lublin: Wydawnictwo Uniwersytetu Marii Curie-Skłodowskiej.

Schwarz, M. (1996). Einführung in die kognitive Linguistik. Tübingen: A. Francke Verlag.

Skowronek, B. (2013). Glottodidaktik und Fremdsprachenunterricht in der Diskussion. Poznań: Wydawnictwo Naukowe Uniwersytetu im. Adama Mickiewicza.

Surkamp, C. (Hrsg.). (2010). Metzler Lexikon. Fremdsprachendidaktik. Stuttgart: Verlag J. B. Metzler.

Szczodrowki, M. (2009). Fremdsprachliche Lehr-Lern-Vorgänge im kodematischen Blickfeld. Gdańsk: Wydawnictwo Uniwersytetu Gdańskiego.

Szeluga, A. (2003). Pragmalinguistische Komponenten der kommunikativen Kompetenz in fremdkultureller Perspektive. In: Zybatow, L.W. (Hrsg.), Linguistik International. Europa der der Sprachen: SprachkompetenzMehrsprachigkeit- Translation. Teil I: Sprache und Gesellschaft. Akten des 35. Linguistischen Kolloguiums in Innsbruck 2000 (S. 411-417). Frankfurt/M: Peter Lang Verlag.

Szeluga, A. (2008). Miejsce dydaktyki języków obcych we współczesnych badaniach językoznawczych. Wybrane aspekty. In: Szeluga, A. (Hrsg.), Dydaktyka języków obcych w świetle współczesnych badań lingwistycznych. (S. 7-17). Włocławek: Wydawnictwo Państwowej Wyższej Szkoły Zawodowej.

Szeluga, A. (2013). Angewandte Fremdsprachendidaktik? Methodologie, Perspektiven und einige Forschungskonzepte aus polnischer Sicht, In: Lipczuk, R., Nerlicki, K. (Hrsg.), Synchronische und diachronische Aspekte der Sprache. 
Sprachwandel-Sprachkontakte-Sprachgebrauch. (S. 245-252). Hamburg: Verlag Dr. Kovac.

Zabrocki, L. (1977). Grundfragen des Deutschunterrichts in fremdsprachiger Umgebung. Poznań: Państwowe Wydawnctwo Naukowe. 